\title{
Frequency Dependence of the Specular and Diffuse Phonon Scattering from Silicon Surfaces
}

\author{
S. Burger, K. Lassmann, and W. Eisenmenger \\ Physikalisches Institut Teil 1, Universität Stuttgart, West Germany
}

(Received May 30, 1985)

Phonon backscattering experiments with polished silicon surfaces show that there is no Kapitza anomaly at frequencies corresponding to the aluminum junction detector threshold $(\sim 80 \mathrm{GHz})$, whereas at higher frequencies the anomalous transmission into liquid helium or solid nitrogen increases (reduced backscattering by the coverage), closely related to the increase of the diffuse scattering at the uncovered surface.

\section{INTRODUCTION}

The ratio of specular to diffuse backscattering of high-frequency phonons from polished surfaces has been investigated by several authors using phonon pulse techniques. Horstman and Wolter ${ }^{1}$ measured the phonon reflection off a (111) silicon- ${ }^{4}$ helium interface at normal incidence geometry by using their heater-bolometer "sandwich." They pointed out the importance of a combination of phonon focusing and diffuse scattering for the interpretation of the observed signal peaks. Using nearly the same geometry Folinsbee and Harrison ${ }^{2}$ found for a (111) silicon- ${ }^{3}$ helium interface that diffusely scattered transverse phonons had reflection coefficients dropping with increasing heater temperature. Marx et al., ${ }^{3}$ using Sn tunnel junctions as phonon detectors (detection above $285 \mathrm{GHz}$ ), found that in the case of silicon, diffuse scattering is dominant for all polishing procedures applied, and that it is strongly reduced by a coverage of the surface with liquid helium or solid nitrogen. Taborek and Goodstein ${ }^{4,5}$ estimated a 50:50 ratio of the two types of scattering for factory-polished $\mathrm{Al}_{2} \mathrm{O}_{3}$ crystals if the scattering surface is in vacuum, whereas the diffuse part is nearly completely reduced when the surface is covered with liquid helium. Therefore it is not astonishing that Northrop and Wolfe ${ }^{6}$ observed only specularly reflected phonons with $\mathrm{Al}_{2} \mathrm{O}_{3}$ crystals immersed into liquid helium. Taborek and Goodstein as well as Northrop and Wolfe used superconductor bolometers 
as phonon detectors, so that a large part of the signal was contributed by low-frequency phonons. Using Al junctions as detectors, no effect of helium coverage has been found if the surfaces are prepared in situ either by cleaving $\mathrm{NaF}^{8}$ or laser annealing silicon. ${ }^{9}$

We have investigated the dependence of surface scattering on phonon frequency and on polishing treatment for $\mathrm{Al}_{2} \mathrm{O}_{3}$ in more detail, ${ }^{7}$ with the following results: At high frequencies ( $\mathrm{Sn}$ detector and/or high heater power) the ratio of diffuse to specular scattering is $4: 3$, and does not depend on the polishing procedure, whereas at low frequencies ( $\mathrm{Al}$ detector, small heater power) the scattering depends on the agent of the last polishing step and may be completely specular.

In this paper we report on an extension of these experiments to silicon. It turns out that at low phonon frequencies (Al detector) complete specular reflection can be obtained by a standard chemical mechanical polish (Syton: an alkaline suspension of colloided silica), whereas at high frequencies diffuse scattering is always dominant, confirming Marx's results. Covering the surface with liquid helium or solid nitrogen again reduces only the diffuse part of the signal. In other words, conventionally polished silicon surfaces may not show the Kapitza anomaly at low frequencies corresponding to an $\mathrm{Al}$ detector, but nevertheless will do so when measured with a Sn junction as detector (high phonon frequencies).

\section{EXPERIMENTAL PROCEDURE}

Sample preparation and measuring arrangement are essentially as described by Marx and Eisenmenger. ${ }^{3}$ After lapping with $9-\mu \mathrm{m}$ diamond the silicon crystals were polished with Syton. The last step of the cleaning procedure was a short HF dip to remove the oxide. After evaporation of the generators and detectors under high-vacuum conditions the crystal was mounted with its reflecting surface onto a UHV-tight vacuum chamber (Fig. 1).

\section{EXPERIMENTAL RESULTS}

The measurements were carried out at about $1.0 \mathrm{~K}$. Information on frequency dependence was obtained by using four types of generator $\rightarrow$ detector setups:

1. Constantan heater $\rightarrow$ tin junction.

2. Constantan heater $\rightarrow$ aluminum junction.

3. Tin junction $\rightarrow$ tin junction.

4. Tin junction $\rightarrow$ aluminum junction. 


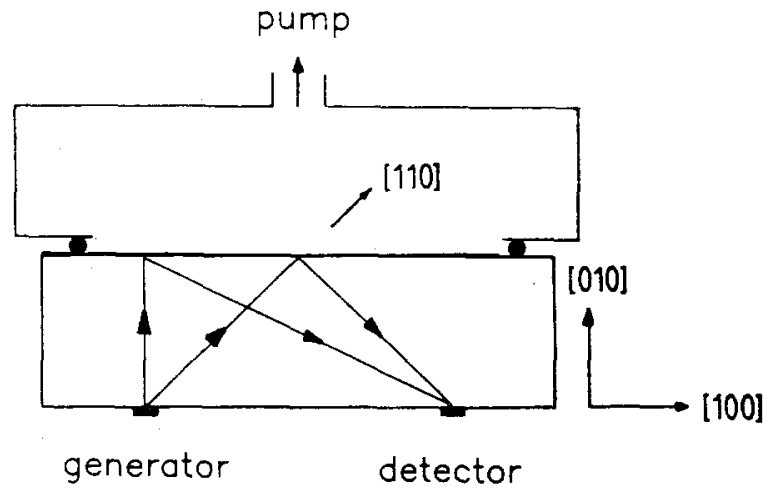

Fig. 1. Experimental arrangement. The sample is pressed with the scattering surface against a UHV chamber. Generator and detector are immersed in liquid helium.

For the interpretation of the results the following facts have to be kept in mind:

1. A Sn junction as generator not only emits high-frequency phonons as determined by the junction bias, but also a large background of lowfrequency phonons due to inelastic scattering at the $\mathrm{Sn} /$ substrate interface. ${ }^{10,11}$

2. A Sn junction as a detector at $1.0 \mathrm{~K}$ has a sharp detection threshold at $1.2 \mathrm{meV}(285 \mathrm{GHz})$. Low-frequency phonons will not be detected.

3. On the other hand, an Al junction as a detector at $1.0 \mathrm{~K}$ has a "soft" detection threshold corresponding to the $\mathrm{Al}$ gap of about $0.3 \mathrm{meV}$ (varying with the oxygen content of the Al films), that is, phonons with energies below the Al gap also will be detected ${ }^{12,13}$ (bolometric detection) through phonon absorption by the large number of quasiparticles excited at this temperature.

4. Since the phonon generators are in contact with liquid helium, the frequency dependence of the Kapitza anomaly ${ }^{13}$ will influence the phonon spectrum emitted into the substrate.

Figure 2 shows experimental results, at frequencies above $285 \mathrm{GHz}$, for the scattering signals from silicon surfaces of very different optical quality with a heater emitter and $\mathrm{Sn}$ junction detector. In the case of Syton polish the surface is optically smooth, whereas with the $0.25-\mu \mathrm{m}$ diamond polish (curve 1) fine scratches can still be seen. Curve 2 corresponds to a surface roughened by lapping with $15-\mu \mathrm{m}$ diamond. All curves look quite similar and correspond to the diffuse reflection pattern obtained by Marx and Eisenmenger, changing only little with varying heater power.

In contrast, using $\mathrm{Al}$ tunnel junctions as detectors, we obtain a strong heater power (i.e., frequency) dependence of the diffuse signal, as shown 


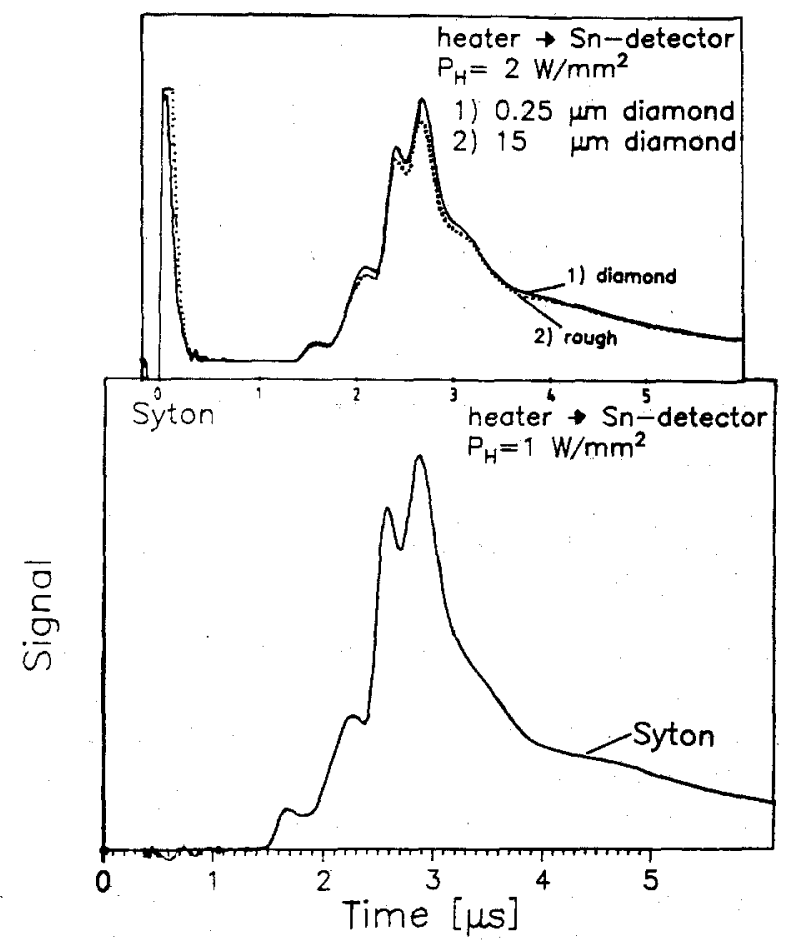

Fig. 2. Detector signal at high frequencies $(\geqslant 285 \mathrm{GHz})$ for various polishing treatments (Syton, $0.25-\mu \mathrm{m}$ diamond, $15-\mu \mathrm{m}$ diamond). The pulse length is 100 nsec.

in Fig. 3 for a Syton polished surface. Here we have plotted the reflection signal for various heater powers as detected by a $0.33-\mathrm{meV} \mathrm{Al} \mathrm{junction.} \mathrm{At}$ the lowest power the signal consists of sharp peaks, which can be attributed to the longitudinal, the mode-converted, and the two transverse specular reflections, followed by multiple specular reflections quite similar to the situation in $\mathrm{Al}_{2} \mathrm{O}_{3} .{ }^{7}$ At higher powers the signal approximates the diffuse pattern (Fig. 2), characterized by an additional diffuse peak, which increases with frequency (see arrow in Fig. 3). The multiple specular reflection peaks are nevertheless visible because of the low-frequency detection of the Al junction.

The effect of covering the reflecting surface with liquid helium or solid nitrogen is quite the same as for $\mathrm{Al}_{2} \mathrm{O}_{3}{ }^{7}$. At the lowest heater power (Fig. 4a) there is practically no influence of the coverage (no Kapitza anomaly), whereas at the highest heater powers (Fig. $4 \mathrm{~b}$ ) there is a strong reduction of the diffuse part of the signal and the remaining signal corresponds very nearly to the specular pattern at low powers (Fig. 3). 


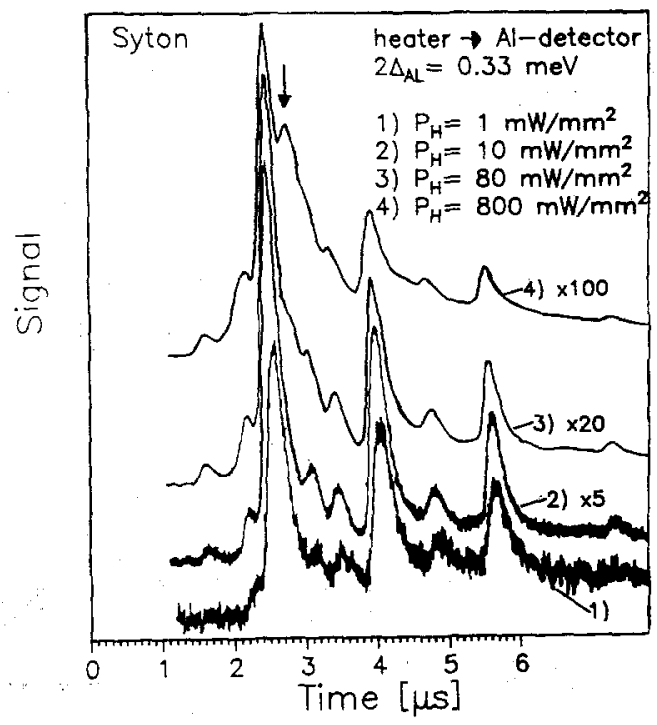

Fig. 3. Heater power dependence of the backscattering signal for a Syton-polished silicon surface and an Al junction as detector. The arrow marks the position of the additional diffuse peak, which appears at higher frequencies (curves 3 and 4).

Figure 5 shows the frequency dependence of a surface polished with $0.25-\mu \mathrm{m}$ diamond instead of the Syton polish (Fig. 3). It is clear that in this case the diffuse scattering is stronger (especially at the highest power level) and at the same time the specular scattering is weaker (as seen from the faster decay of the multiple echoes). Correspondingly, the reduction of the signal by helium coverage is much stronger at the highest power level (Fig. 6b) than in the case of Syton polish, whereas at the lowest power (Fig. $6 a)$ there is also a very small reduction. The strong reduction at high powers shows that most of the diffusely scattered phonons have reached the surface. In contrast, for the roughened surface of curve 2 in Fig. 2 the influence of helium coverage was very small. From this one might conclude that the phonons have not reached the surface, but have been scattered off a subsurface damage layer.

It must, however, be kept in mind that diffuse scattering and small reduction by coverage may also occur for a special roughness without subsurface damage: If by some surface preparation one obtains smooth facets of statistical orientation, the resulting scattering pattern would correspond to the diffuse case, but the reduction by helium coverage should be small, because the elementary scattering process is specular in character. 

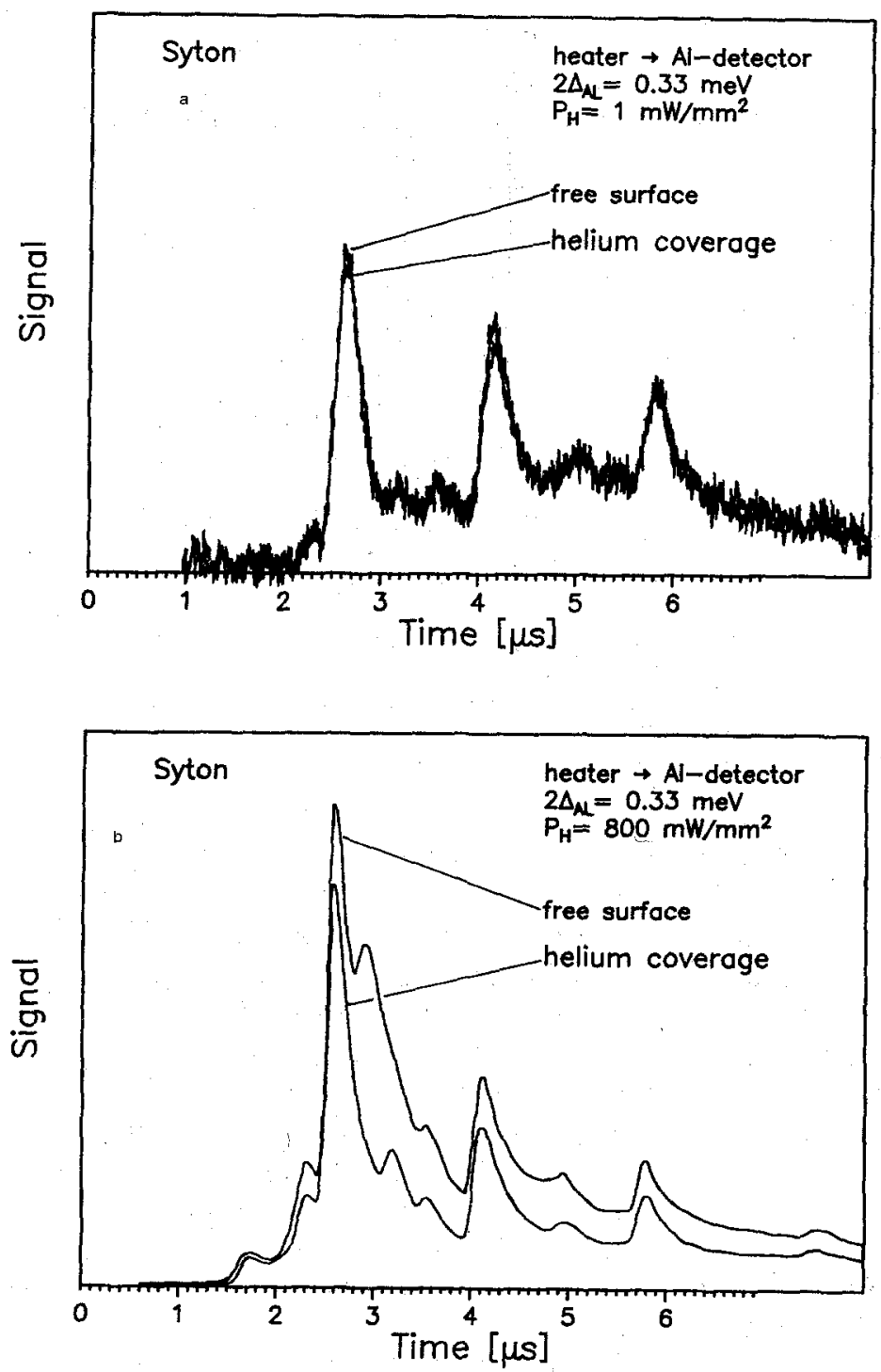

Fig. 4. Detector signal for a Syton-polished surface without and with helium coverage. (a) Low heater power: the two curves are nearly identical (no Kapitza anomaly). (b) High heater power: nearly complete reduction of the diffuse scattering. 


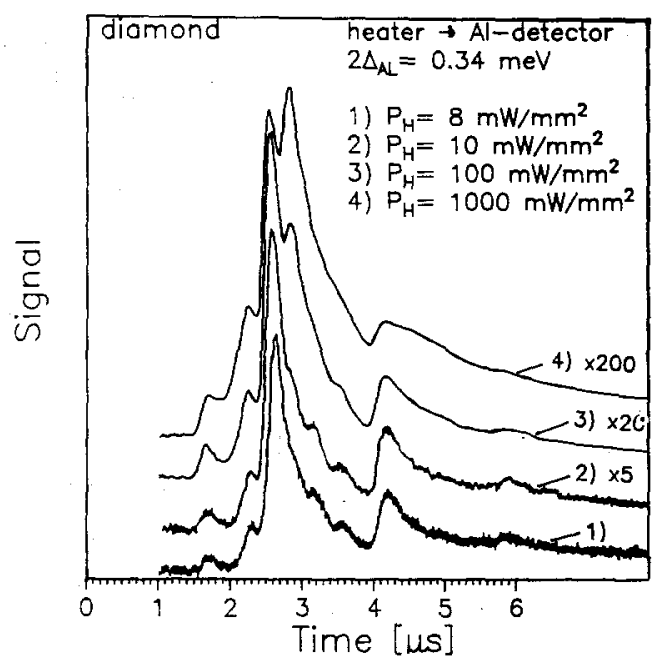

Fig. 5. Heater power dependence of the backscattering signal for a $0.25-\mu \mathrm{m}$ diamond-polished surface and Al junction detector. The diffuse scattering is increased as compared to Syton polish.

This remark points to the necessity of a better characterization of the surface roughness as related to the anomalous transmission.

These examples show that the heater generator $\rightarrow \mathrm{Al}$ junction detector combination together with the crystal geometry used is a sensitive means to probe the quality of real surfaces, whereas $\mathrm{Sn}$ junction detectors do not distinguish between different conventional surface preparations. ${ }^{3,7}$ Recently, however, it has been observed ${ }^{14}$ that in situ laser-annealed silicon surfaces also show a strong increase of the specular reflection with Sn junction detectors.

On the other hand, Sn junctions as phonon generators may emit a large background of low-frequency phonons together with a relatively small portion of quasimonochromatic phonons with energies determined by the junction bias. ${ }^{10,11}$ This is demonstrated by Fig. 7a, showing that the signal of the $\mathrm{Al}$ detector is determined by the specular reflection of low-frequency phonons, although the $\mathrm{Sn}$ generator is driven at a bias of $2 \Delta / e$ or $4 \Delta / e$. However, in a Sn junction emitter/Sn junction detector arrangement one obtains the diffuse pattern (Fig. $7 \mathrm{~b}$ ) because the Sn detector is only sensitive to the high-frequency part of the phonons.

Therefore, in order to make reliable statements on phonon frequencies with tunnel junction generators a modulation technique is necessary. However, with this technique time-resolved reflection measurements are 

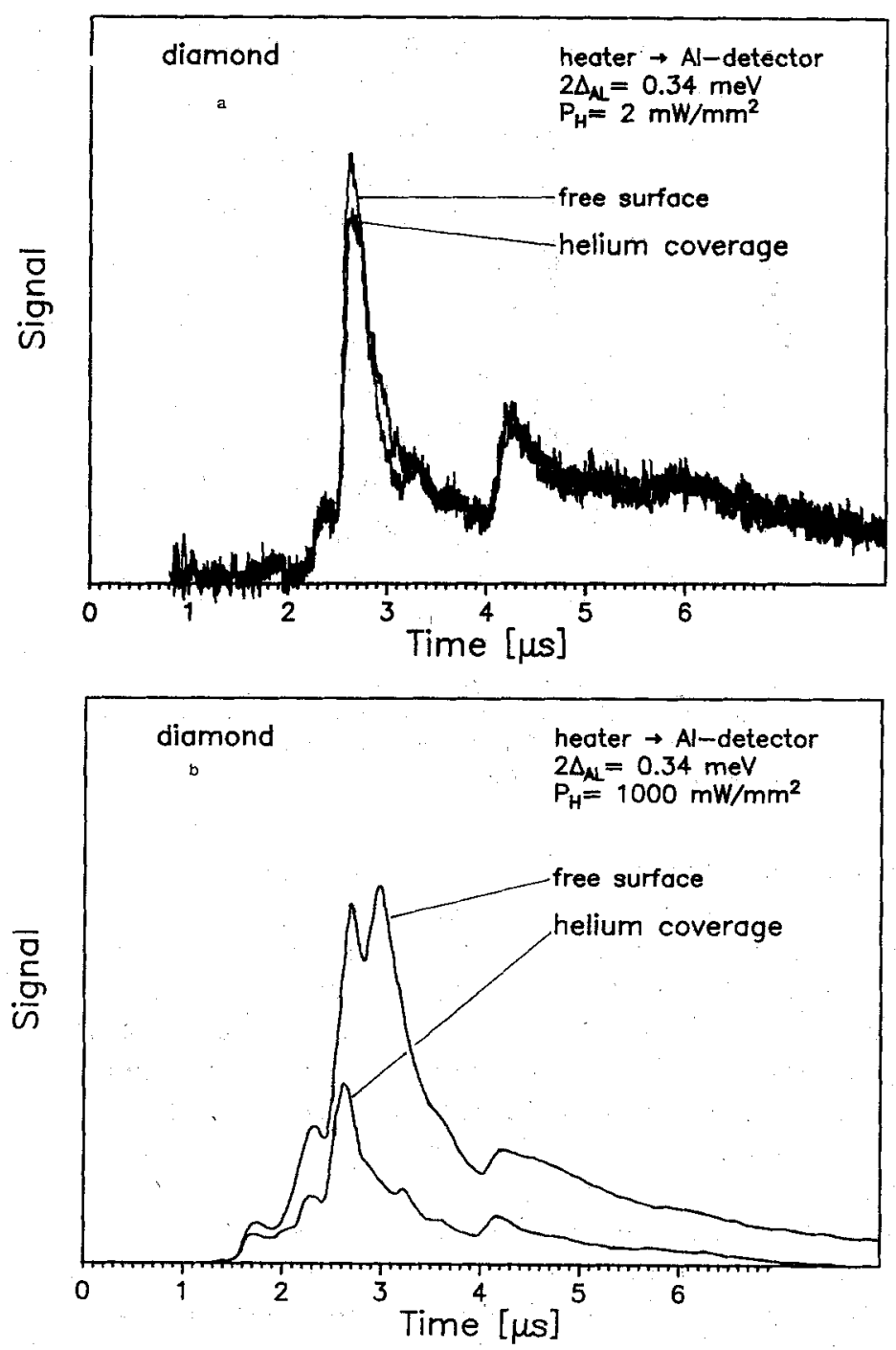

Fig. 6. Influence of helium coverage on the diamond polished surface for (a) low and (b) high heater power.

rather difficult because of the geometrical spread of the phonon intensities. Instead, we have plotted in Fig. 8 the differential Al signal vs. Sn generator current (modulated $\mathrm{cw}$, no time resolution in this case), which demonstrates the bolometric detection of our $\mathrm{Al}$ junctions: there is a signal rise at a generator current corresponding to $2 \Delta_{\text {detector }}$ relaxation phonons (arrow), preceded, however, by a comparable rise for generator voltages between 

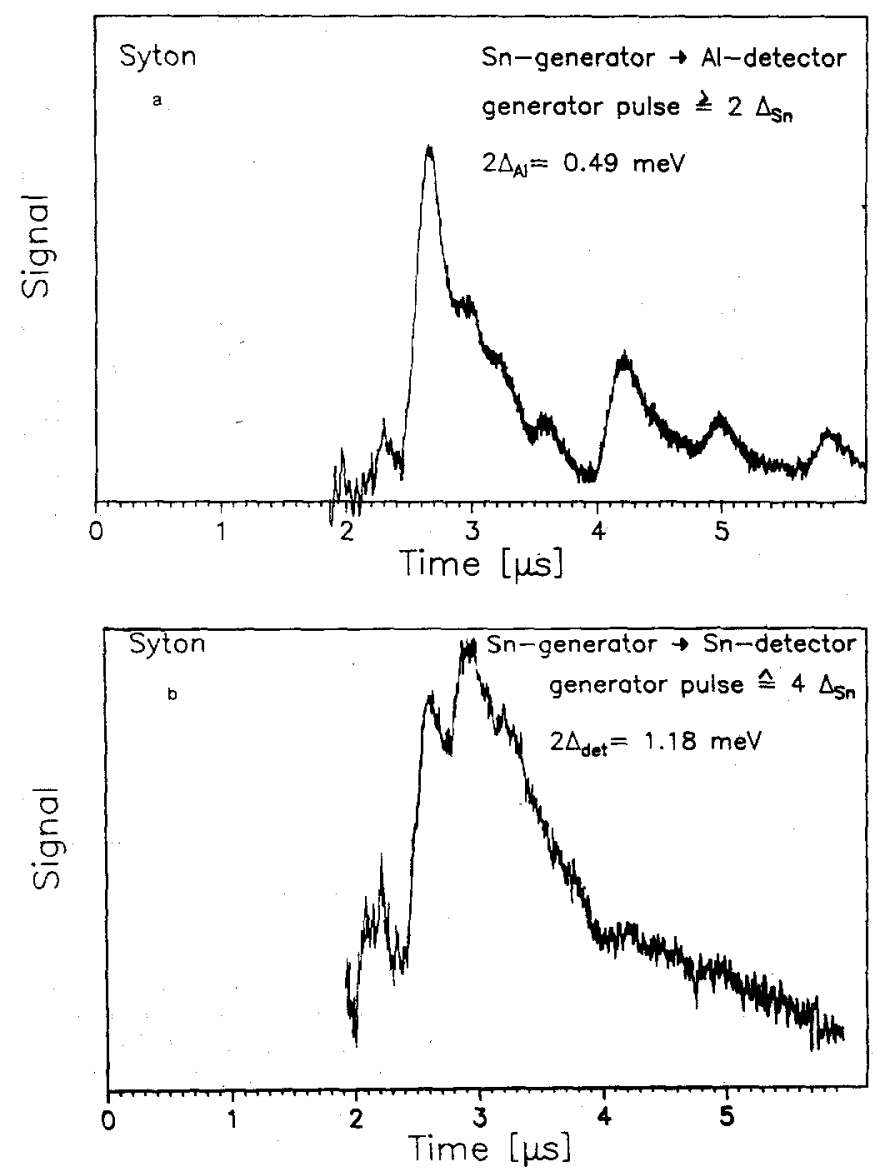

Fig. 7. (a) Time-resotved signal of an Al junction detector. The Sn junction generator is driven at a bias of $\sim 2 \Delta / e$. (b) Time-resolved signal of a $\mathrm{Sn}$ junction detector. The Sn junction generator is driven at a bias of $4 \Delta / e$.

$2 \Delta_{\mathrm{Sn}}$ and $2 \Delta_{\mathrm{Sn}}+2 \Delta_{\mathrm{Al}}$ where relaxation phonons with energies below the detector gap are produced.

The decay of the signal above about $0.35 \mathrm{meV}$ is due to the enhanced anomalous transmission ${ }^{13}$ from the $\mathrm{Sn}$ generator directly into the adjacent liquid helium. Curve 1 is the signal off the uncovered scattering surface; curve 2 is for the helium-covered surface. Curve 3 corresponds to curve 2, but is normalized to curve 1 at $0.35 \mathrm{meV}$ for comparison to show a rather important feature: the threshold for anomalous transmission for the silicon/helium interface is the same as for the $\mathrm{Sn} /$ helium and $\mathrm{SiO} /$ helium 


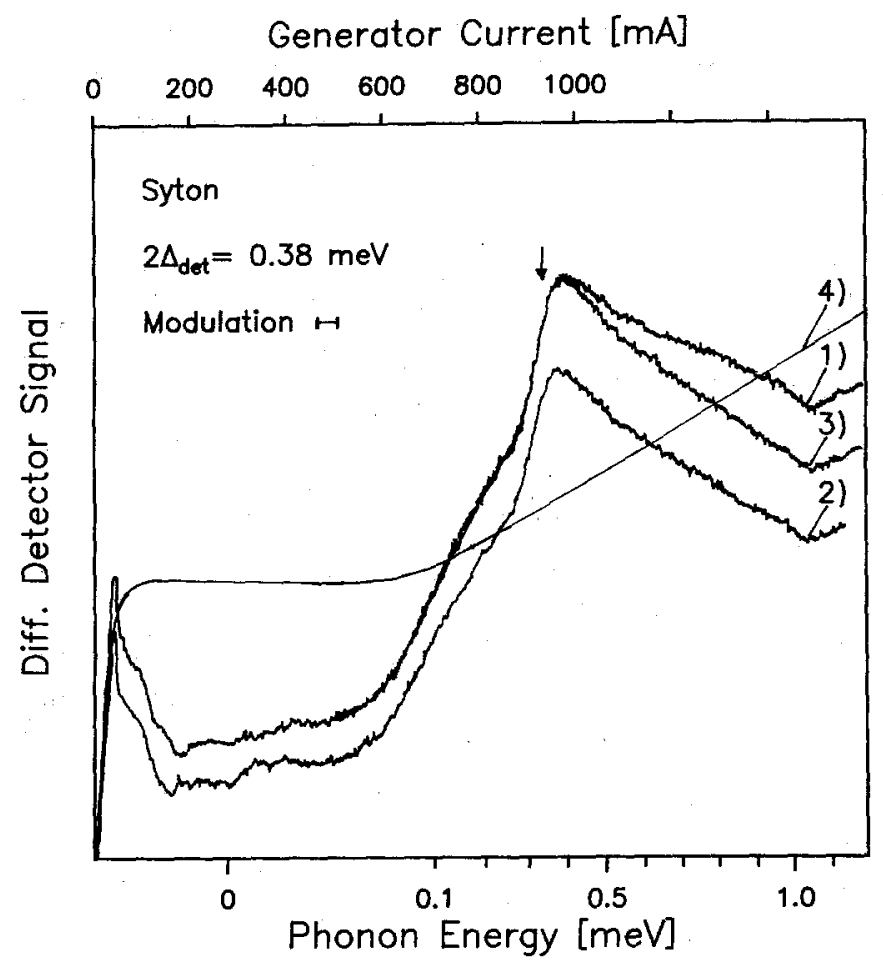

Fig. 8. Differential phonon signal of an Al junction detector as a function of the Sn generator current. The arrow marks the position of the detector gap. curve (1) Free backscattering surface, (2) helium-covered surface, (3) same as curve 2 , but normalized to curve 1 at $0.35 \mathrm{meV}$, (4) $I-V$ curve of the $S n$ generator $\left(2 \Delta_{\text {gen }}=1.18 \mathrm{meV}\right)$.

interfaces. ${ }^{12,13}$ This is another indication that the frequency threshold does not depend on the material of the transmitting solid, but is related to the properties of the covering medium together with some surface irregularity or contamination.

\section{CONCLUSION}

We have studied the reflection of high-frequency acoustic phonons at silicon surfaces prepared with various polishing procedures. We used either normal conducting heaters or superconducting tunnel junctions as phonon generators. The detectors were tunnel junctions with different detection thresholds.

For silicon surfaces polished to an optical finish by standard methods (Syton) the scattering is completely specular for frequencies below about 
$80 \mathrm{GHz}$. In the frequency range above around $80 \mathrm{GHz}$ there is a steep increase of diffuse scattering. Coverage of the surface by liquid helium or solid nitrogen reduces nearly completely the diffuse part of the backscattering signal. At low frequencies there is no reduction of the signal by helium coverage (no Kapitza anomaly), though the silicon surface is only conventionally polished. Frequency-resolved measurements for helium coverage reveal the same frequency threshold for reduction as has been found previously for the anomalous transmission ${ }^{12,13}$ across $\mathrm{Sn} /$ helium and $\mathrm{SiO} /$ helium interfaces.

Experiments with diamond-polished surfaces (grain sizes 0.25 or $15 \mu \mathrm{m}$ ) show that surface roughness is responsible at least for part of the diffuse scattering. Remembering that Koblinger et al., ${ }^{13}$ comparing ${ }^{4} \mathrm{He}$ and ${ }^{3} \mathrm{He}$, found a shift of the frequency threshold of the anomalous transmission from it might be speculated that it is the combination of surface roughness with a certain van der Waals-type coverage that leads to a specific transmission threshold independent of the substrate material. This corresponds to geometrically induced surface states at the solid/helium boundary. However, in the case of sapphire surfaces polished with Syton to a smoothness on the scale of the resolution of a standard TEM, i.e., about $10 \mathrm{~nm}$, we have found ${ }^{7}$ strong diffuse scattering already at low frequencies, which was also completely reduced by helium coverage. This points more to the chemical than to the geometrical state of the surface as responsible for both the diffuse scattering as well as the anomalous transmission.

Our results show that the reported combination of time- and frequencyresolved phonon reflection measurements is a promising tool for studying the properties of real surfaces.

\section{ACKNOWLEDGMENTS}

We thank G. Untereiner for the preparation of the sample surfaces and W. Burger for many discussions. Financial support of the Deutsche Forschungsgemeinschaft is gratefully acknowledged.

\section{REFERENCES}

1. R. E. Horstman and J. Wolter, Phys. Lett. A62, 297 (1977).

2. J. T. Folinsbee and J. P. Harrison, J. Low Temp. Phys. 32, 469 (1978).

3. D. Marx, J. Buck, K. Lassman, and W. Eisenmenger, J. Phys. C6, 1015 (1978); D. Marx and W. Eisenmenger, Phys. Lett. A82, 291 (1981); D. Marx and W. Eisenmenger, Z. Phys. B48, 277 (1982).

4. P. Taborek and D. Goodstein, J. Phys. C 12, 4737 (1979).

5. P. Taborek, and D. Goodstein, Phys. Rev. B 22, 1550 (1980).

6. G. A. Northrop and J. P. Wolfe, Phys. Rev. Lett. 52, 2156 (1984). 
7. S. Burger, W. Eisenmenger, and K. Lassmann, in Proceedings LT17 (North-Holland, Amsterdam, 1984); in Fifth General Conference of the Condensed Matter Division of the EPS (1985), Vol. 9A.

8. J. Weber, W. Sandmann, W. Dietsche, and H. Kinder, Phys. Rev. Lett. 40, 1469 (1978).

9. H. Basso, W. Dietsche, and H. Kinder, in Phonon Scattering in Condensed Matter, W. Eisenmenger et al., eds. (Springer Series in Solid-State Sciences 51, 1984).

10. H. J. Trumpp and W. Eisenmenger, $Z$. Phys. $B$ 28, 159 (1977).

11. P. Berberich and H. Kinder, $J$. Phys. (Paris) 42, C6-374 (1981).

12. O. Koblinger, Thesis, Universität Stuttgart (1983).

13. O. Koblinger, U. Heim, M. Welte, and W. Eisenmenger, Phys. Rev. Lett. 51, 284 (1983).

14. E. Mok, S. Burger, S. Döttinger, and W. Eisenmenger, in Fifth General Conference of the Condensed Matter Division of the EPS (1985), Vol. 9A. 